\title{
Impact of serum interleukin 22 as a biomarker for the differential use of molecular targeted drugs in psoriatic arthritis: A retrospective study
}

Ippei Miyagawa

University of Occupational and environmental Health, Japan

Shingo Nakayamada

University of Occupational and environmental Health, Japan

Masanobu Ueno

University of Occupational and environmental Health, Japan

Yusuke Miyazaki

University of Occupational and environmental Health, Japan

Shigeru Iwata

University of Occupational and environmental Health, Japan

Satoshi Kubo

University of Occupational and environmental Health, Japan

Koshiro Sonomoto

University of Occupational and environmental Health, Japan

Junpei Anan

University of Occupational and environmental Health, Japan

Naoaki Ohkubo

University of Occupational and environmental Health, Japan

Yoshino Inoue

University of Occupational and environmental Health, Japan

Yoshiya Tanaka ( $\nabla$ tanaka@med.uoeh-u.ac.jp)

University of Occupational and environmental Health, Japan

\section{Research Article}

Keywords: Psoriatic arthritis, precision medicine, interleukin 22, tumor necrosis factor inhibitors, interleukin 17 inhibitors

Posted Date: January 19th, 2022

DOI: https://doi.org/10.21203/rs.3.rs-1265931/v1 
License: (c) (i) This work is licensed under a Creative Commons Attribution 4.0 International License. Read Full License

Version of Record: A version of this preprint was published at Arthritis Research \&amp; Therapy on April 15th, 2022. See the published version at https://doi.org/10.1186/s13075-022-02771-4. 


\section{Abstract}

Background. We explored whether serum cytokines could be used as biomarkers for optimal use of tumor necrosis factor inhibitors (TNF-i) and interleukin (IL) 17 inhibitors (IL-17-i) in patients with psoriatic arthritis (PsA).

Methods. In cohort 1 (47 patients treated with IL-17-i [n=23] or TNF-i [n=24] for $\geq 1$ year), we identified serum cytokines that predicted the achievement of Disease Activity in Psoriatic Arthritis- remission (DAPSA-REM), Psoriasis Area and Severity Index (PASI) 90 and Minimal Disease Activity after 1 year of TNF-i or IL-17-i therapy. Subsequently, we developed treatment strategies based on the identified cytokines; initiation of IL-17-i therapy in patients with low IL-22 concentrations (IL-22 $<0.61376 \mathrm{pg} / \mathrm{ml}$ ) and TNF-i therapy in patients with high IL-22 concentrations $(0.61376<\mathrm{IL}-22 \mathrm{pg} / \mathrm{ml})$. In cohort 2 (34 patients), treatment responses were compared between the strategic treatment group $(n=17)$, which was treated based on the treatment strategies, and the mismatched treatment group $(n=17)$ to verify the validity of the treatment strategies developed using serum cytokines as biomarkers.

Results. In cohort 1, serum IL-22 concentration was identified as a predictor of DAPSA-remission after 1 year of IL-17-i therapy. Regarding treatment strategies, we selected TNF-i for patients with high IL-22 concentrations and IL-17-i for those with low IL-22 concentrations. There were no significant differences in the baseline characteristics between the strategic and mismatched treatment groups. Regarding treatment effects, disease activity significantly improved at 1 year in both groups. Upon comparison of the treatment effects, the rate of achieving DAPSA-REM and Minimal Disease Activity at month 12 was significantly higher in the strategic treatment group.

Conclusions. We verified that serum IL-22 can be used as a biomarker for the proper selection of TNF-i and IL-17-i.

\section{Background}

Psoriatic arthritis (PsA) is a typical complication of psoriasis (PS) that often causes irreversible destruction and dysfunction of the peripheral joints or the spine. Various cytokines, such as interferon (IFN)-y, interleukin (IL)-12, IL-23, IL-17, IL-6, and tumor necrosis factor (TNF) a, play an important role in the pathogenesis of PS and PsA. The European League Against Rheumatism (EULAR) recommends using molecular targeted drugs for the management of PsA with pharmacological therapies (2019 update) to treat PSA and PS, such as TNF-inhibitors (TNF-i), IL-17-inhibitors (IL-17-i), IL-12/23-inhibitors (IL-12/23-i), Janus kinase inhibitors (JAK-i), and phosphodiesterase 4 inhibitors (PDE4-i) (1). However, some issues remain. For example, some patients are resistant to these drugs and require changes in their treatment strategies. Although drugs target different molecules, no optimal drug selection method has been established. Studies directly comparing TNF-i and IL-17-i have shown that these drugs are equally effective $(2,3)$. The establishment of an optimal selection method for these drugs can contribute to better patient outcomes. 
Recently, efforts have been made to establish precision medicine that aims to improve patient outcomes in systemic autoimmune diseases with high heterogeneity. To implement precision medicine, stratification of patients and selective use of molecular targeted drugs is recommended (4). Indeed, we attempted precision medicine based on the stratification of patients by lymphocyte phenotyping in patient peripheral blood and found that the proportion of $\mathrm{T}$ follicular helper cells in the peripheral blood of patients with rheumatoid arthritis was an independent predictor of favorable responses to abatacept therapy (5). In addition, we demonstrated that excessive B cell differentiation is associated with treatment resistance in anti-neutrophil cytoplasmic antibody-associated vasculitis, and that rituximab is more effective in patients with circulating B cell abnormalities (6). Furthermore, we showed that the rate of achieving low disease activity as assessed by the Simplified Disease Activity Index (SDAI) after 6 months of treatment was significantly higher in 46 patients with PsA, in whom biological therapy was strategically initiated based on phenotyping of peripheral blood lymphocytes in PsA compared to 38 patients who were conventionally treated with biological drugs based on the 2011 and 2015 EULAR recommendations (7). We reported the possibility of stratification of patients by phenotyping peripheral blood lymphocytes and precision medicine based on the selective use of molecular targeted drugs in systemic autoimmune diseases, such as PsA. However, since phenotyping of peripheral blood lymphocytes is complex and feasible at a limited number of institutions, the development of simple methods using biomarkers to stratify patients and simple treatment strategies based on such methods is needed to promote precision medicine in a real-world clinical setting.

\section{Methods}

\section{Patients and Clinical Measurement}

This retrospective study aimed to identify predictors of treatment responses to biological drugs in 47 patients with PSA treated with TNF-i or IL-17-i for $\geq 1$ year while focusing on the serum cytokine concentrations at treatment initiation. This study included two cohorts. Cohort 1 consisted of 47 patients diagnosed with PsA based on the Classification Criteria for Psoriatic Arthritis at multiple institutions affiliated to our university hospital, the key station (FIRST registry). The patients were treated with TNF-i (24 cases) or IL-17-i (23 cases) for $\geq 1$ year between February 2015-when IL-17-i (secukinumab) was approved for reimbursement under the National Health Insurance system for the first time in Japan-and 2018. Cohort 2 (validation cohort) consisted of patients who started treatment with TNF-i (17 cases) or IL17-i (18 cases) between 2019 and 2020 and were treated for $\geq 1$ year. In both cohorts, the biological drugs were selected based on shared decision-making between the attending physicians and patients. Disease activity was assessed up to 1 year after initiation of the biological therapies in both cohorts. The treatment effects of the biological drugs were assessed using the disease activity in psoriatic arthritis (DAPSA), psoriasis area and severity index (PASI) and Minimal Disease Activity. Meanwhile, seven patients in cohort 1 and five patients in cohort 2 with PASI at 0 at baseline were excluded from the calculation of PASI response rates (PASI75 and PASI90). In cohort 2, the serum cytokine concentrations of all the patients were measured after $\geq 1$ year treatment. The treatment was discontinued due to adverse events or insufficient efficacy in one patient each in both cohorts. In these patients, the last 
observation carried forward (LOCF) method was used to impute the missing data with the latest available data.

\section{Establishment of treatment strategy based on serum cytokine concentration}

Treatment strategies were established based on the data on serum cytokine concentrations in cohort 1 (Study 1). In the 24 patients treated with TNF-i and the 23 patients treated with IL-17-i, univariate logistic analyses were separately performed with achievement of DAPSA remission (REM), PASI90 and Minimal disease Activity at 1 year as an objective variable. In addition, each serum cytokine concentration was used as an explanatory variable to identify serum cytokines that predicted the achievement of DAPSAREM, PASI90 and Minimal disease activity at 1 year separately in the TNF-i-treated and IL-17-i-treated groups. Furthermore, we developed treatment strategies based on the IL-22 cut-off value of 0.61376 (sensitivity, 81.8\%; specificity, 91.7\%; area under the curve, 0.848), which was identified as a predictor for DAPSA-REM after 1 year of IL-17-i therapy. Based on these strategies, IL-17-i therapy was initiated in patients with IL-22 concentrations lower than the cut-off value at treatment initiation, and TNF-i therapy was initiated in patients with higher IL-22 concentrations (Figure 1A).

\section{Validation of the treatment strategy in cohort 2}

The efficacy of the treatment strategies developed in cohort 1 was validated in cohort 2 (validation cohort) (Study 2). The treatment effects over 1 year were retrospectively compared between the strategic treatment group, which included 24 patients whose treatment matched the treatment strategies developed based on the results obtained from cohort 1 , and the mismatched treatment group, which included 17 patients. Specifically, the strategic treatment group included patients with low IL-22 concentrations who were treated with IL-17-i and those with high IL-22 concentrations who were treated with TNF-i, whereas the mismatched treatment group included patients with low IL-22 concentrations who were treated with TNF-i and those with high IL-22 concentrations who were treated with IL-17-i. The efficacy of the biological drugs over 1 year was compared between these groups to assess the validity of the treatment strategies (Figure 1B).

\section{Serum cytokine measurement}

In cohorts 1 and 2, serum cytokine (IFN-y, IL-6, TNF-a, IL-17A, IL-21, IL-22, and IL-23) concentrations were measured. Similarly, these concentrations were also measured in the healthy control $(\mathrm{HC})$ group matched for age and sex. In all patients in cohorts 1 and 2, serum cytokine concentrations at the initiation of the biological therapies were measured after I year treatment were completed. The MESO SCALE DISCOVERY S-PLEX Human IL-17A kit (\#K15067L-1, Meso Scale Diagnostics, LLC, Rockville, MD, USA) was used to measure IL-17A (fg/ml), and the U-PLEX Biomarker Group 1 (\#K151C3S-1, Meso Scale Diagnostics, LLC, Rockville, MD, USA) was used to measure IFN-y (pg/ml), IL-6 (pg/ml), TNF-a (pg/ml), IL-21 (pg/ml), IL-22 $(\mathrm{pg} / \mathrm{ml})$, and IL-23 (pg/ml) (8).

\section{Statistical analysis}


Data are expressed as mean \pm standard deviation, median (interquartile range [IQR]), or number (\%). Differences between groups were compared using the Mann-Whitney U test, Kruskal-Wallis test with Dunn's correction for multiple comparisons or the chi-square test. The Wilcoxon signed rank test was used to identify statistically significant differences between the baseline data and those measured at months 6 and 12. In cohort 1, univariate logistic analyses were performed using achievement of DAPSAREM, PASI90 and Minimal Disease Activity at 1 year as an objective variable and serum concentrations of IFN- $y$, IL-6, TNF-a, IL-17A, IL-21, IL-22, and IL-23 as explanatory variables to identify serum cytokines that predicted achievement of DAPSA-REM, PASI90 and Minimal Disease Activity at 1 year separately in the TNF-i-treated and IL-17-i-treated groups. All reported P-values were two-sided. The level of significance was set at $p<0.05$. All analyses were conducted using JMP Pro version 15 (SAS Institute Inc., Cary, NC, USA) and GraphPad Prism 9 (GraphPad Software, San Diego, CA, USA).

\section{Results}

\section{Identification of serum cytokines that predict bDMARDs treatment response}

Supplemental Table S1 shows the baseline characteristics and serum cytokine concentrations at the initiation of the bDMARD therapies in cohort 1 (TNF-i: 24 cases, IL-17-i: 23 cases). Although serum IFN- $y$, IL-6, TNF, and IL-21 concentrations tended to be higher in patients with PsA compared to that in the HC group, no statistically significant differences were observed. Alternatively, serum IL-17 concentrations were significantly higher in patients with PsA compared to that in the HC group (Supplementary Figure S1).

In this non-randomized retrospective study, statistically significant differences were found in the prevalence of complications (diabetes mellitus) and serum IL-17A (Supplemental Table S1); meanwhile, disease activity did not differ between the TNF-i-treated and IL-17-i-treated groups. One year after the initiation of bDMARD therapy, disease activity was significantly improved. DAPSA-REM was achieved in 24 patients (51.3\%), PASI90 in 26 of 40 patients (65.0\%), and Minimal Disease Activity in 33 patients (70.2 \%) (Supplementary Table S2). In addition, there was no difference in efficacy at 1 year between TNF-i therapy and IL-17-i therapy (supplementary Table S3).

Then, in the TNF-i-treated group (24 cases) and IL-17-i-treated group (23 cases), we performed univariate logistic analyses with achievement of DAPSA-REM, PASI90 and Minimal Disease Activity as objective variables and serum cytokine concentrations as explanatory variables. Serum IL-22 concentrations were identified as a factor contributing to the achievement of DAPSA-REM in the IL-17-i-treated group. However, no baseline serum cytokines were identified as factors contributing to achievement of DAPSA-REM in the TNF-i-treated group or achievement of PASI90 and Minimal Disease Activity in either group (Supplementary Tables S4). We compared baseline serum cytokine concentrations among patients who achieved DAPSA-REM (12 cases), those who did not (12 cases), in the TNF-i-treated group and corresponding patients in the IL-17-i-treated group (11 and 12 cases, respectively). The results 
showed that the baseline serum IL-22 concentrations were significantly lower in patients who achieved DAPSA-REM than in those who did not achieve DAPSA-REM in the IL-17-i-treated group (Figure 2).

\section{Establishment of treatment strategy based on serum cytokine concentration}

Using a cut-off value of IL-22 0.61376 (sensitivity, 81.8\%; specificity, 91.7\%; area under the curve, 0.848) determined by a receiver operating characteristic analysis, we stratified 24 patients in the TNF-i-treated group and 23 patients in the IL-17-i-treated group into the IL-22 high group $(0.61376<$ IL-22) and the IL-22 low group (IL-22 <0.61376) (Supplementary Tables S5 and S6). Subsequently, the treatment courses over 1 year were retrospectively compared. No significant differences were observed in treatment responses at 1 year (Supplementary Tables S5). Alternatively, in the IL-17-i-treated group, although the baseline characteristics showed a significantly higher proportion of patients with concomitant use of methotrexate (MTX) at treatment initiation in the IL-22 low group (Supplementary Tables S6), the rate of achieving DAPSA-REM and Minimal Disease Activity after 1 year of IL-17-i therapy was significantly higher in the IL22 low group (Table 1). Regarding achievement of PASI75/90, no statistically significant differences were observed between the IL-22 high and IL-22 low groups in either the TNF-i-treated or IL-17-i-treated group.

In addition, serum cytokine concentrations were compared among the IL-22 high (25 cases), IL-22 low (22 cases), and HC groups. Serum IL-17 concentrations were significantly higher in both the IL-22 high and IL22 low groups than in the HC group, whereas no significant difference was observed between the IL-22 high and IL-22 low groups. Alternatively, the serum TNF-a concentrations did not significantly differ between the IL-22 low and HC groups; however, they were significantly higher in the IL-22 high group than in the $\mathrm{HC}$ and IL-22 low groups (Figure 3). Based on these results, we created treatment strategies using TNF-i and IL-17-i based on serum cytokine (IL-22) concentrations, that is, initiation of IL-17-i therapy in patients with low IL-22 concentrations and TNF-i therapy in patients with high IL-22 concentrations.

\section{Validation of the treatment strategy in cohort 2}

To validate the efficacy of the treatment strategies using TNF-i and IL-17-i based on serum IL-22 concentrations, we retrospectively compared the efficacy of the biological drugs at 1 year between the following groups in cohort 2 . The strategic treatment group (17 cases) included patients with low IL-22 concentrations who were treated with IL-17-i and those with high IL-22 concentrations who were treated with TNF-i. The mismatched treatment group (17 cases) included patients with low IL-22 concentrations who were treated with TNF-i and those with high IL-22 concentrations who were treated with IL-17-i. Table 2 shows the baseline characteristics of the strategic treatment group (17 cases) and the mismatched treatment group (17 cases). No statistically significant differences were observed between the two groups in baseline characteristics at the initiation of bDMARD therapy. There were no statistically significant differences between the groups in the concomitant use of MTX or the proportion of patients receiving bDMARDs (Table 2).

Table 3 also shows the treatment responses over 1 year. After initiation of bDMARD therapy, tender joint counts, swollen joint counts, C-reactive protein (CRP), DAPSA, and PASI were significantly improved in 
both groups (Supplementary Figure S2). When the treatment responses over 1 year were compared between the two groups, the rate of achieving Minimal Disease Activity at month 6, DAPSA-REM and Minimal Disease Activity at month 12 was significantly higher in the strategic treatment group. There were no statistically significant differences in the rates of achieving PASI75 or PASI90 at month 6 or 12 between the two groups (Table 3 ).

\section{Discussion}

This report is the first to demonstrate the possibility of optimizing the selection between TNF-i and IL-17-i based on serum IL-22 concentrations.

PsA is a disease with very high heterogeneity, and its clinical symptoms vary among patients. Thus, we focused on serum cytokine concentrations as biomarkers that could contribute to simple and proper drug selection. In addition, we previously reported that drug selection based on phenotyping of peripheral blood lymphocytes leads to more frequent achievement of low disease activity as assessed by the SDAI (7). In the present study, we aimed to develop treatment strategies that would lead to achievement of DAPSA-REM, PASI90 or Minimal Disease Activity and more effective control of disease activity.

Since treatment was selected based on shared decision-making between the attending physicians and the patients in this non-randomized study, there were statistically significant differences in the prevalence of comorbid diabetes mellitus and serum IL-17A concentrations measured after the end of the observation period in cohort 1 (Supplementary Table S1). However, we continued the analyses described below, because we did not intend to directly compare the efficacy of TNF-i and IL-17-i. Instead, we aimed to identify cytokines predictive of responses to each of the TNF-i and IL-17-i therapies and to develop new treatment strategies based on the identified cytokines.

Univariate logistic analyses identified serum IL-22 concentrations as a factor contributing to the achievement of DAPSA-REM in IL-17-i therapy (Supplementary Table S4).

Patients in cohort 1 were stratified based on serum IL-22 concentrations, and the efficacy of IL-17-i and TNF-i therapies was evaluated. The results showed that significantly more patients with low IL-22 concentrations who were treated with IL-17-i achieved DAPSA-REM and Minimal Disease Activity (Table 1). Meanwhile, a comparison of the baseline characteristics showed significant differences in the concomitant use of MTX (Supplementary Table S6). Although we considered that low IL-22 concentrations may reflect the low inflammatory state due to MTX, no statistically significant differences were observed in the baseline values of CRP, or serum IL-22 concentrations between the 29 patients with concomitant use of MTX and the 18 patients without in cohort 1 (Supplementary Figure S3). Similarly, there was no significant difference in serum IL-17 concentrations between these two groups with and without concomitant use of MTX. Since serum concentrations of IL-17 and IL-22 did not correlate with CRP (Supplementary Figure S4), the levels of CRP and the concomitant use of MTX seemed unlikely to reflect the serum concentrations of IL-17 and IL-22. Thus, we focused on IL-22 concentrations as a simple biomarker for the proper selection of TNF-i and IL-17-i to develop treatment strategies. 
Moreover, no factors contributing to the achievement of PASI90 were identified for either TNF-i or IL-17-i therapy (Supplementary Table S4), and no differences were observed in efficacy in terms of PASI during treatment (Table 1 and 3). This may have been due to the fact that the PASI was already favorable at baseline.

Similar to helper T (Th) 17 cells, Th22 expresses chemokine receptor (CCR) 4 and CCR6. In addition, IL-22 is a cytokine produced by Th22 cells, a relatively new Th cell subset that is positive for CCR10 and expresses the aryl hydrocarbon receptor as the master transcription factor. In addition, an increase in IL22-producing $\mathrm{CD} 4^{+} \mathrm{T}$ cells in the peripheral blood, as well as an increase in IL-17-producing CD $4^{+} \mathrm{T}$ cells is characteristic of PS and PsA. In addition, serum IL-22 concentrations have been reported to be characteristically and significantly higher in PsA than in PS (9). In a study using an in vitro experimental system with human naïve T cells, we demonstrated that the addition of IL-1 $\beta$, TNF-a, and IL- 6 to T cell receptors stimulation induces $\mathrm{CD} 3^{+} \mathrm{CD} 4^{+} \mathrm{CCR} 4^{+} \mathrm{CCR} 6^{+} \mathrm{CCR} 10^{+} \mathrm{Th} 22$ cells that selectively produce IL-22 (10). Moreover, when serum cytokine concentrations were compared among the HC, IL-22 high, and IL-22 low groups in the present study, serum IL-17 concentrations were significantly higher in both the IL-22 high and IL-22 low groups than in the HC group, whereas no significant difference was observed between the IL-22 high and IL-22 low groups. Alternatively, serum TNF-a concentrations were significantly higher only in the IL-22 high group than in the HC and IL-22 low groups (Figure 3). As described above, the differentiation of Th22 cells is dependent of TNF-a; however, the differentiation of Th17 cells is not (10). Therefore, we assumed that cell populations characterized by IL-17 production, such as Th17 cells and innate lymphoid cells (ILCs), may be mainly involved in the pathogenesis of patients with low IL-22 concentrations, and that, in addition to Th17 cells and ILCs, Th22 cells, which are characterized by the production of IL-17 and IL-22 and induced by cytokines including TNF-a, may be involved in the pathogenesis of patients with high IL-22 concentrations. Based on this assumption, we developed treatment strategies for initiating TNF-i therapy in patients with high IL-22 concentrations and IL-17-i therapy in patients with low IL-22 concentrations.

In cohort 2 (validation cohort), we compared treatment responses over 1 year between the strategic and mismatched treatment groups. The results showed that the rate of achieving DAPSA-REM and Minimal Disease Activity at 1 year was significantly higher in the strategic treatment group (Table 3 ). As we hypothesized, it was suggested that patients with PsA may include two types of patients. In the first type of patients, Th17 cells and ILCs, which are involved in IL-17 production, are mainly involved in pathogenesis, which is consistent with the conventional assumption. In the second type of patients, Th22 cells, which are involved in IL-22 production, are also involved in the pathogenesis of this disease. Future studies on the correlation between patients with high IL-22 concentrations and Th22 cell subsets in peripheral blood and on changes in serum concentrations of IL-22 and Th22 cells due to treatment are expected to advance the elucidation of the pathology with a focus on the Th22-IL-22 correlation.

This study excluded patients using IL-12/23 (p40) inhibitors, because no clinical studies have demonstrated that IL-12/23 (p40) inhibitors are comparable to TNF-i or IL-17-i. This study focused on the proper selection of only TNF-i and IL-17-i. Moreover, patients using IL-23 (p19) inhibitors, which were 
approved in Japan only shortly before this study, were also excluded due to an insufficient number of patients. Based on our hypothesis that there are patients whose main pathogenic factors are Th17 cells and ILCs, which are involved in IL-17 production, and patients in whom Th22 cells involved in IL-22 production are also involved in the pathogenesis, IL-23 (p19) inhibitors may be more effective in the former. In addition, the use of JAK-i has recently expanded to include the treatment of PsA $(11,12)$. Since JAK-i is expected to inhibit the differentiation of Th17 cells as well as Th22 cells, it appears to be a potential drug that is highly effective for a wide spectrum of PsA.

\section{Conclusions}

This study demonstrated that serum IL-22 can be used as a simple biomarker for the proper selection of TNF-i and IL-17-i. However, the sample size was as small at 81 cases. Further studies that include a larger sample size are needed to develop more precise treatment strategies and to evaluate their effects in detail.

\section{Abbreviations}

TNF-i: tumor necrosis factor inhibitors interleukin

IL-17-i: interleukin17 inhibitors

PsA: psoriatic arthritis

PS: psoriasis

IFN-y: interferon $y$

IL: interleukin

JAK-i: Janus kinase inhibitors

PDE4-i: phosphodiesterase 4 inhibitors

DAPSA: Disease activity in psoriatic arthrits

PASI: psoriasis area and severity index

REM: remission

HC: healthy control

CCR: chemokine receptor

\section{Declarations}




\section{Ethics approval and consent to participate}

Ethical approval was obtained from the University of Occupational and Environmental Health, Japan Ethics Committee (approval number \#04-23), based on the Helsinki Declaration,linformed consent was obtained from all patients of the FIRST registry.

Consent for publication: Not applicable.

Availability of data and materials: Not applicable

Conflict of interest: Y. Tanaka has received speaking fees and/or honoraria from Daiichi-Sankyo, Eli Lilly, Novartis, YL Biologics, Bristol-Myers, Eisai, Chugai, Abbvie, Astellas, Pfizer, Sanofi, Asahi-kasei, GSK, Mitsubishi-Tanabe, Gilead, and Janssen; research grants from Abbvie, Mitsubishi-Tanabe, Chugai, AsahiKasei, Eisai, Takeda, and Daiichi-Sankyo; and consultant fees from Eli Lilly, Daiichi-Sankyo, Taisho, Ayumi, Sanofi, GSK, and Abbvie.

S. Nakayamada received consulting fees, speaking fees, and/or honoraria from Bristol-Myers, Pfizer, GlaxoSmithKline, Sanofi, Astellas, Asahi-kasei, and Boehringer Ingelheim and research grants from Mitsubishi-Tanabe and Novartis.

Funding: This work was supported in part by Research on rare and intractable diseases and Research Grant-In-Aid for Scientific Research by the Ministry of Health, Labour and Welfare of Japan, the Ministry of Education, Culture, Sports, Science and Technology of Japan; the Japan Agency for Medical Research and Development; PATNER fellowship; and the University of Occupational and Environmental Health, Japan and UOEH Grant for Advanced Research(\#19K17919).

\section{Authors' contributions}

IM contributed to the study design, overall review, and writing of the manuscript, and the other authors were involved in the performance of the study and review of the manuscript. MU, KS, JA, NO, and YI contributed to serum cytokine measurements. YT, SI, SN, and MY participated in the study design and coordination. All authors have read and approved the final version of the manuscript.

\section{Acknowledgements}

The authors thank the study participants, without whom this study could not have been accomplished, and all medical staff at all participating institutions for providing the data, especially Ms. Hiroko Yoshida, Ms. Youko Saitou, Ms. Machiko Mitsuiki and Ms. Ayumi Maruyama for the excellent data management in the FIRST registry. The authors thank Ms. M. Hirahara for providing excellent technical assistance. We also thank Dr Kazuyoshi Saito at Tobata General Hospital; Dr Kentaro Hanami and Dr Shunsuke Fukuyo at Wakamatsu Hospital of the University of Occupational and Environmental Health; Dr Keisuke Nakatsuka at Fukuoka Yutaka Hospital, and all staff members at Kitakyushu General Hospital and 
Shimonoseki Saiseikai Hospital. Nakama Municipal Hospital, and Steel Memorial Yawata Hospital for their engagement in data collection of the FIRST registry.

\section{References}

1. Gossec L, Baraliakos X, Kerschbaumer A, de Wit M, Mclnnes I, Dougados M, et al. EULAR recommendations for the management of psoriatic arthritis with pharmacological therapies: 2019 update. Ann Rheum Dis 2020,79.700-712

2. Smolen JS, Mease P, Tahir H, Schulze-Koops H, de la Torre I, Li L, Hojnik M, et al. Multicentre, randomised, open-label, parallel-group study evaluating the efficacy and safety of ixekizumab versus adalimumab in patients with psoriatic arthritis naïve to biological disease-modifying antirheumatic drug: final results by week 52. Ann Rheum Dis 2020,79.1310-1319

3. McInnes IB, Behrens F, Mease PJ, Kavanaugh A, Ritchlin C, Nash P, et al. Secukinumab versus adalimumab for treatment of active psoriatic arthritis (EXCEED): a double-blind, parallel-group, randomised, active-controlled, phase $3 \mathrm{~b}$ trial Lancet 2020,395.1496-1505

4. Miyagawa I, Kubo S, Tanaka Y. A wide perspective of targeted therapies for precision medicine in autoimmune diseases. Expert Rev Precis Med Drug Dev. 2020 https://doi.org/10.1080/23808993.2020.1804867

5. Nakayamada S, Kubo S, Yoshikawa M, Miyazaki Y, Yunoue N, Iwata S, et al. . Differential effects of biological DMARDs on peripheral immune cell phenotypes in patients with rheumatoid arthritis. Rheumatology(Oxford) 2018;1;57:164-174. doi: 10.1093/rheumatology/kex012.

6. Miyazaki Y, Nakayamada S, Kubo S, Ishikawa Y, Yoshikawa M, Sakata K, et al. Favorable efficacy of rituximab in ANCA associated vasculitis patients with excessive B cell differentiation. Arthritis Res Ther. 2020:22:141.

7. Miyagawa I, Nakayamada S, Nakano K, Kubo S, Iwata S, Miyazaki Y, et al. . Precision medicine using different biological DMARDs based on characteristic phenotypes of peripheral T helper cells in psoriatic arthritis.Rheumatology (Oxford) 2019:1;58:336-344. doi: 10.1093/rheumatology/key069.

8. Dabitao D, Margolick JB, Lopez J, Bream JH. Multiplex measurement of proinflammatory cytokines in human serum: comparison of the Meso Scale Discovery electrochemiluminescence assay and the Cytometric Bead Array. J Immunol Methods 2011,372.71-77

9. Benham H, Norris P, Goodall J, Wechalekar MD, FitzGerald O, Szentpetery A et al. Th17 and Th22 cells in psoriatic arthritis and psoriasis. Arthritis Res Ther. 2012,26.R136.

10. Miyazaki Y, Nakayamada S, Kubo S, Nakano K, Iwata S, Miyagawa I, et al. Th22 Cells Promote Osteoclast Differentiation via Production of IL-22 in Rheumatoid Arthritis. Front Immunol. 2018:10;9: 2901. doi:10.3389/fimmu.2018.02901.

11. Mease P, Hall S, FitzGerald O, van der Heijde D, Merola JF, Avila-Zapata F, et al. Tofacitinib or Adalimumab versus Placebo for Psoriatic Arthritis. N Engl J Med. 2017,377.1537-1550 
12. Mclnnes IB, Anderson JK, Magrey M, Merola JF, Liu Y, Kishimoto M, et al. Trial of Upadacitinib and Adalimumab for Psoriatic Arthritis N Engl J Med 2021,384,1227-1239

\section{Tables}

Table 1. Comparison of 1 year treatment response between IL-22 high and low group

\begin{tabular}{llll} 
Variables & IL-22 low & IL-22 high & p-value \\
\hline [TNF-i treated group[ & $\mathrm{n}=12$ & $\mathrm{n}-12$ & \\
\hline DAPSA-REM & $8(66.7)$ & $5(41.6)$ & 0.4136 \\
\hline DAPSA-LDA & $9(75.0)$ & $10(83.3)$ & 1.0000 \\
\hline Minimal disease activity & $8(66.7)$ & $9(75.0)$ & 1.0000 \\
\hline PASI 75 & $7 / 11(63.6)$ & $5 / 11(45.4)$ & 0.6699 \\
\hline PASI 90 & $9 / 11(81.8)$ & $6 / 11(54.5)$ & 0.3615 \\
\hline - & & & \\
\hline DIL-17-i treated group[ & $\mathrm{n}=10$ & $\mathrm{n}=13$ & \\
\hline DAPSA-REM & $9(90)$ & $2(15.3)$ & $\mathbf{0 . 0 0 0 6 *}$ \\
\hline DAPSA-LDA & $10(100)$ & $12(92.3 \%)$ & 1.0000 \\
\hline Minimal disease activity & $10(100)$ & $6(46.1)$ & $\mathbf{0 . 0 0 7 5 *}$ \\
\hline PASI 75 & $7 / 9(77.8)$ & $7 / 9(77.8)$ & 1.0000 \\
\hline PASI 90 & $7 / 9(77.8)$ & $7 / 9(77.8)$ & 1.0000
\end{tabular}

Data are expressed as number (\%); TNF-i, TNF inhibitors; IL-17-i, IL-17-inhibitors; DAPSA, disease activity in psoriatic arthritis; LDA, low disease activity; REM, remission; PASI, psoriasis area, and severity index ${ }^{*} \mathrm{p}<0.05$, by chi-square test

Table 2. Baseline characteristics of strategic treatment group and un-matched treatment group 


\begin{tabular}{llll} 
Variables & Strategic treatment $(\mathbf{n}=17)$ & mismatched $(\mathbf{n}=17)$ & $\mathrm{p}$-value \\
\hline Age (years) & $53.2 \pm 16.0$ & $58.4 \pm 10.8$ & 0.5016 \\
\hline Male, $\mathbf{n}(\%)$ & $12(70.5)$ & $6(35.3)$ & 0.0084 \\
\hline Disease Duration (months) & & & \\
\hline PSO & $108(42,288)$ & $216(10,258)$ & 0.9176 \\
\hline PsA & $42(13.5,116.5)$ & $43(16.5,144)$ & 0.7960 \\
\hline Bio naive & $12(70.5)$ & $12(70.5)$ & 1.0000 \\
\hline Concomitant MTX use & $12(70.6)$ & $10(58.8)$ & 0.7270 \\
\hline bDMARDS initiated & & & \\
\hline IL-17-i /TNF-i & $6(35.2) / 11(64.7)$ & $9(52.9) / 8(47.0)$ & 0.4905 \\
\hline Disease activity & & & \\
\hline TJC & $7(5,8.5)$ & $8(3.5,14)$ & 0.4164 \\
\hline SJC & $6(4,12.5)$ & $5(2.5,7)$ & 0.1453 \\
\hline CRP (mg/dl) & $0.99(0.18,2.14)$ & $0.54(0.075,1.71)$ & 0.2347 \\
\hline DAPSA & $24.1(19.2,33.7)$ & $31.2(20.9,39.8)$ & 0.1848 \\
\hline PASI & $3.1(0.5,5.75)$ & $1.6(0.4,6.3)$ & 0.7823
\end{tabular}

Data are expressed as mean \pm standard deviation, median (interquartile range [IQR]) or number (\%).

TNF-i: TNF inhibitors; IL-17-i: IL-17-inhibitors; TJC: tender joint counts 68; SJC: swollen joint counts 66; DAPSA, disease activity in psoriatic arthritis; PASI: psoriasis area and severity index * $p<0.05$, by MannWhitney U test or chi-square test

Table 3. Comparison of 1 year treatment response between strategic treatment group and unmatched treatment group 


\section{Month 6}

\begin{tabular}{|c|c|c|c|}
\hline$\Delta \mathrm{TJC}(\mathrm{M} 6-\mathrm{BL})$ & $-6(-8,-4.5)$ & $-5(-11,-2.5)$ & 0.9172 \\
\hline$\Delta \mathrm{SJC}(\mathrm{M} 6-\mathrm{BL})$ & $-4(-7,-2)$ & $-4(-10.5,-2)$ & 0.8086 \\
\hline$\triangle \mathrm{DAPSA}(\mathrm{M} 6-\mathrm{BL})$ & $-18.6(-27.3,-13.7)$ & $-18.4(-32.4,-13.3)$ & 0.9314 \\
\hline \% Decrease-SDAI & $-77.7(-89.9,-71.4)$ & $-74.0(-82.3,-53.2$ & 0.1432 \\
\hline Proportion of REM, $\mathbf{n}(\%)$ & $7(41.1)$ & $3(17.6)$ & 0.2587 \\
\hline Proportion of REM/LDA, $n$ (\%) & 17(100) & $14(82.3)$ & 0.1136 \\
\hline Minimal disease activity & $12(70.6)$ & $4(25.3)$ & $0.0075^{*}$ \\
\hline \% Improvement-PASI & $86.0(53.1,100)$ & $100(17.6,100)$ & 0.9809 \\
\hline PASI90, n (\%) & $7 / 15(46.7)$ & $9 / 14(64.2)$ & 0.9469 \\
\hline PASI75, n (\%) & $7 / 15(46.7)$ & $9 / 14(64.2)$ & 0.9469 \\
\hline
\end{tabular}

\section{Month 12}

\begin{tabular}{|c|c|c|c|}
\hline$\Delta \mathrm{TJC}(\mathrm{M} 12-\mathrm{BL})$ & $-6(-8.5,-3)$ & $-5(-11.5,-2)$ & 0.9175 \\
\hline$\triangle \mathrm{SJC}(\mathrm{M} 12-\mathrm{BL})$ & $-4(-6,-1.5)$ & $-5(-11.5,-2)$ & 0.4365 \\
\hline$\triangle \mathrm{DAPSA}$ & $-18.4(-27.3,-12.9)$ & $-19.45(-33.6,-10.5)$ & 0.8228 \\
\hline$\%$ Decrease-DAPSA (M12) & $-87.4(-97.1,-75.4)$ & $-72.0(-89.2,-44.7)$ & 0.0605 \\
\hline Proportion of REM, $n(\%)$ & $10(58.8)$ & $4(25.3)$ & $0.0399 *$ \\
\hline Proportion of REM/LDA, $n(\%)$ & $15(88.2)$ & 13(76.4) & 0.3281 \\
\hline Minimal disease activity & $14(82.3)$ & $7(41.2)$ & $0.0162^{\star}$ \\
\hline \% Improvement-PASI & $92.7(51.7,100)$ & $100(72.7,100)$ & 0.3794 \\
\hline PASI90, n (\%) & $9 / 15(60)$ & 10/14(71.4) & 0.8502 \\
\hline PASI75, n (\%) & $9 / 15(60)$ & $11 / 14(78.5)$ & 0.9320 \\
\hline
\end{tabular}

Data are expressed as median (interquartile range [IQR] ) or number (\%).

TNF-i: TNF inhibitors; IL-17-i: IL-17-inhibitors; TJC: tender joint counts 68; SJC: swollen joint counts 66; DAPSA: disease activity in psoriatic arthritis; LDA: low disease activity; REM: remission; PASI: psoriasis area and severity index, ${ }^{\star} \mathrm{p}<0.05$, by Mann-Whitney $U$ test or chi-square test. 
(A) Study l: Establishment of treatment strategy based on serum cytokine concentration

\begin{tabular}{|c|c|}
\hline \multicolumn{2}{|l|}{ Cohort $1(n=47)$} \\
\hline TNF-i $(n=24)$ & \\
\hline$\overline{\mathrm{IFX} / \mathrm{CZP}}$ & $: n=1$ \\
\hline $\mathrm{ADA}$ & $: n=22$ \\
\hline
\end{tabular}

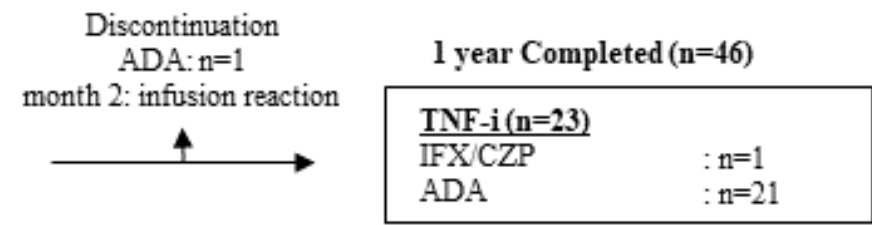

\begin{tabular}{|ll}
\hline$\underline{\operatorname{IL17}}-\mathbf{i}(\mathbf{n}=\mathbf{2 3})$ & \\
SEC & $: \mathrm{n}=18$ \\
IXE & $: \mathrm{n}=5$ \\
\hline
\end{tabular}

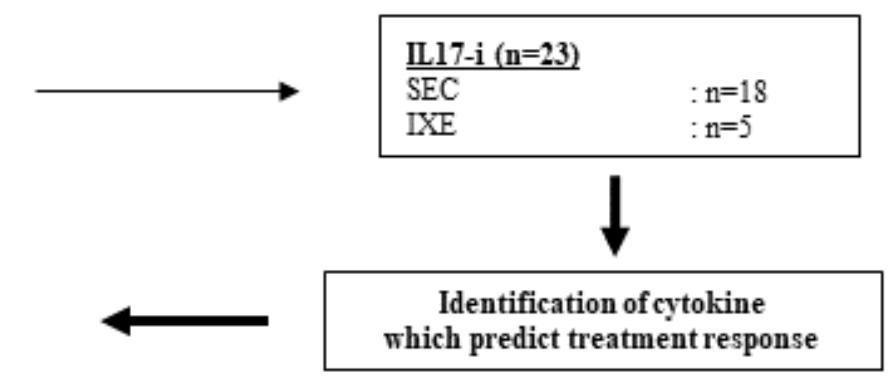

Establishment of treat strategy

based on serum IL-22 concentration

high : TNF-i/Low : ILl7-i

(B) Study 2: Validation of treatment strategy

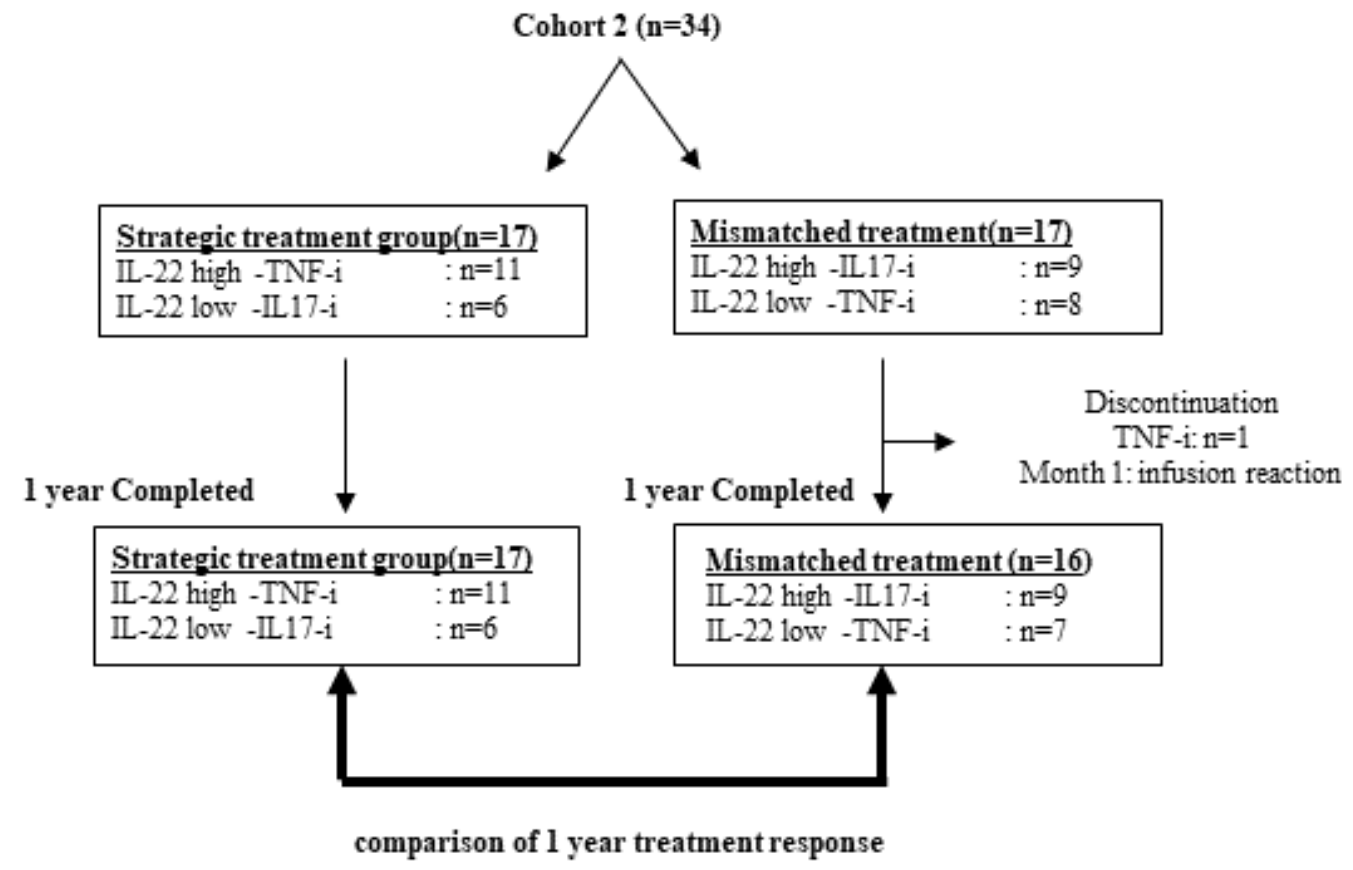

\section{Figure 1}

Study design 
(A)IL17-i-DAPSA-REM or not

IFN- $\gamma(\mathrm{pg} / \mathrm{ml}) \quad \mathrm{IL}-6(\mathrm{pg} / \mathrm{ml}) \quad$ TNF- $\alpha(\mathrm{pg} / \mathrm{ml}) \quad$ IL-17A(fg/ml) $\quad$ IL-2l(pg/ml) $\quad$ IL-22(pg/ml) $\quad$ IL-23(pg/ml)

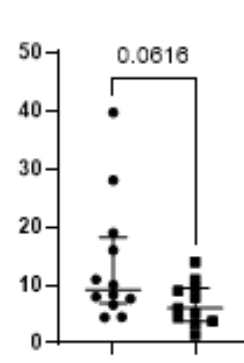

non REM

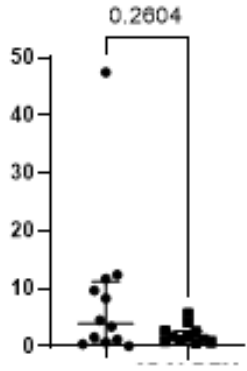

non REM

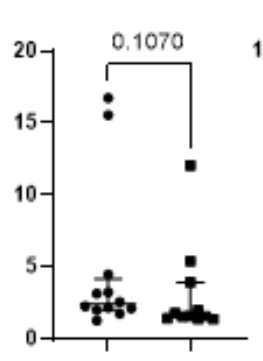

non REM

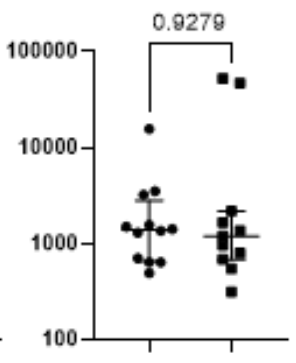

non REM

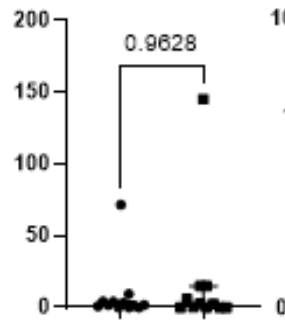

non REM

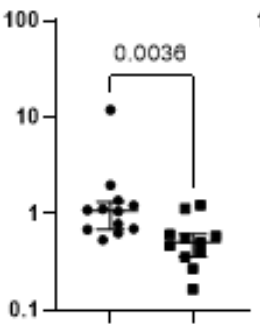

non REM

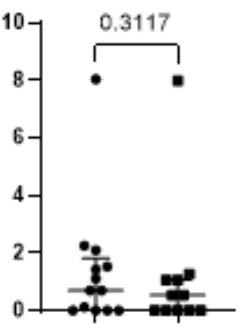

non REM

$9.20(6.85,18.2) \quad 3.99(0.85,11.2)$

$2.42(2.03,4.17) \quad 1390.5(656.7,2813.8) \quad 1.81(0.23,4.17) 1.08(0.69,1.34) \quad 0.90(0.02,1.95)$

$6.02(3.77,9.48) \quad 1.68(0.89,2.67)$

$1.59(1.43,3.94) \quad 1196.5(684.3,2179.2) \quad 2.47(0,14.8)$

$0.50(0.35,0.61)$

$0.51(0,1.06)$

\section{(B) TNF-i-DAPSA-REM or not}

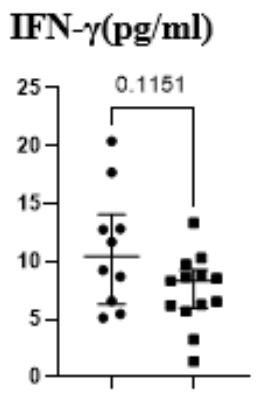

non REM

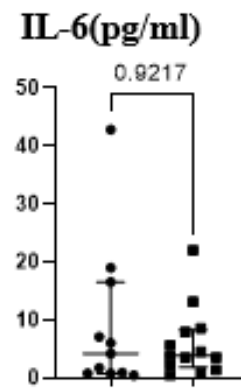

non REM
TNF- $\alpha(p g / m l)$

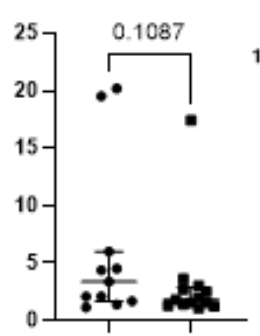

non REM

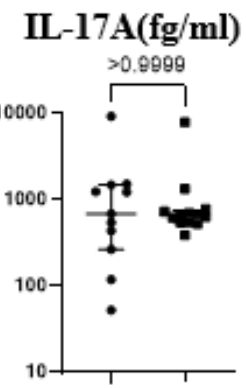

non REM

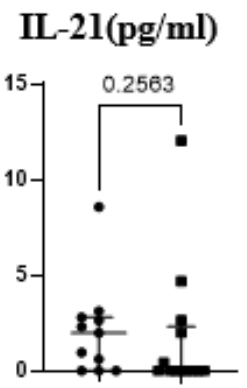

non REM
IL-22(pg/ml)

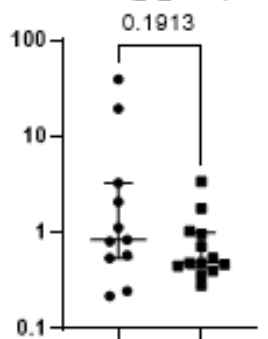

non REM
IL-23(pg/ml)

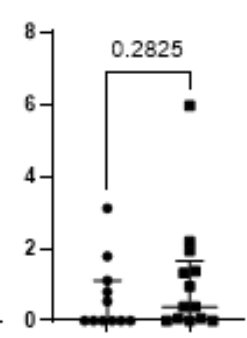

non REM

$11.6(6.58,17.7)$ $4.25(0.85,16.6) \quad 3.39(1.70,6.01)$

$685.9(265.3,1480.0)$

$1.97(0,2.79)$

$0.84(0.54,3.29) \quad 0(0,1.11)$

$8.31(5.94,9.28)$

Figure 2

Baseline serum cytokine concentrations in patients who achieved DAPSA-REM and not

Data are presented as median (interquartile range). (A)Differences in serum cytokine concentrations in patients treated with interleukin 17 inhibitors (IL-17-i) between patients with DAPSA-REM $(n=11)$ and those without (non) ( $n=12)$. (B)Comparison of serum cytokine concentrations in patients treated with tumor necrosis factor inhibitors (TNF-i) between patients with SDAI-REM $(n=12)$ and those without (non) $(\mathrm{n}=12) .{ }^{*} p<0.05$, Mann-Whitney U test 
IFN- $\gamma(\mathrm{pg} / \mathrm{ml})$

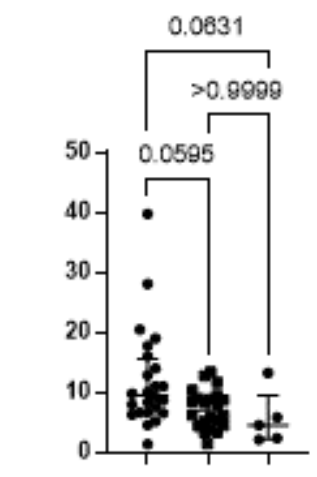

$\begin{array}{lll}9.58 & 7.00 \quad 4.50\end{array}$

$(6.58,15.5)(4.43,9.10)(2.23,4.50)$

IL-17A(fg/ml)

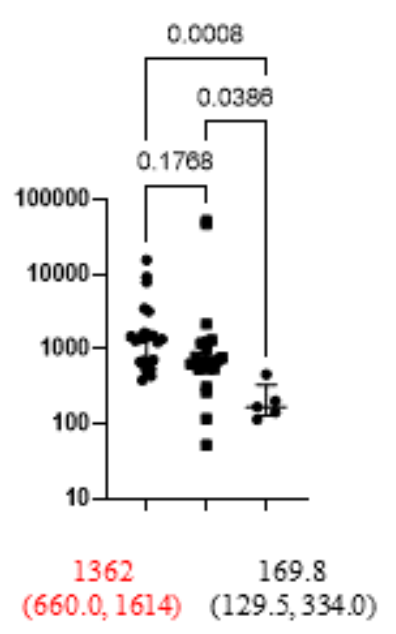

678.8

$(544.9,678.8)$
IL-6(pg/ml)

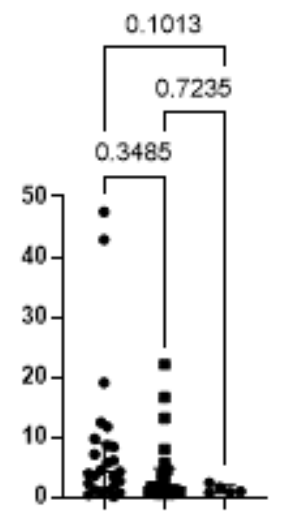

$\begin{array}{ccc}4.25 & 1.63 & 0.98 \\ (1.52,9.16) & (0.88,4.86) & (0.72,2.02)\end{array}$

IL-2l(pg/ml)

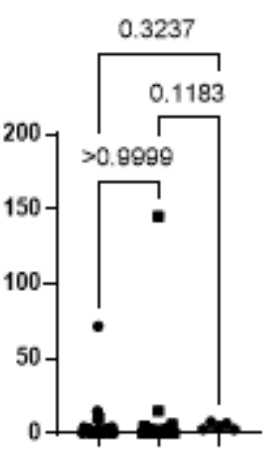

$\begin{array}{lll}1.81 & 0.31 & 3.74\end{array}$

$(0,4.13)(0,2.83)(2.71,7.09)$
TNF- $\alpha(\mathrm{pg} / \mathrm{ml})$

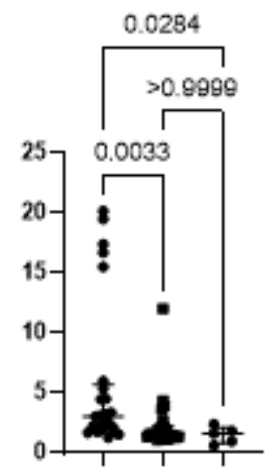

$\begin{array}{lll}3.05 & 1.57 \quad 1.62\end{array}$

$(2.00,5.71)(1.37,2.31)(0.80,2.13)$

\section{IL-23(pg/ml)}

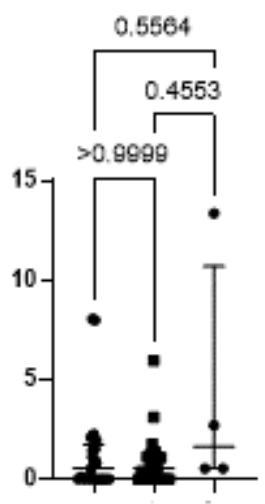

$\begin{array}{lll}0.54 & 0.54 & 1.62\end{array}$

$(0,1.73)(0,1.28)(0.54,10.7)$

Figure 3

Comparison of cytokine concentration among the IL-22 high group, IL-22 low group, and healthy controls

Data are presented as median (interquartile range). Serum cytokine concentrations were compared among patients with high interleukin (IL) 22 concentrations $(n=25)$, patients with low IL-22 concentrations $(\mathrm{n}=22)$, and healthy controls $(\mathrm{HC})(\mathrm{n}=5) .{ }^{*} p<0.05$, by Kruskal-Wallis test with Dunn's correction.

\section{Supplementary Files}


This is a list of supplementary files associated with this preprint. Click to download.

- SupplementaryTableS1..docx

- SupplementaryTableS2..docx

- SupplementaryTableS3..docx

- SupplementaryTableS4..docx

- SupplementaryTableS5..docx

- SupplementaryTableS6..docx

- SupplementaryFigureS1..pptx

- SupplementaryFigureS2..pptx

- SupplementaryFigureS3..pptx

- SupplementaryFigureS4..pptx 